\title{
Polymicrobial Keratitis-Importance of Detecting and Treating More Than One Organism
}

\author{
Uma Sridhar1, Parul Jain1, Jyoti Batra1, Neelam Sapra² \\ ${ }^{1}$ Cornea Services ICARE Eye Hospital, Noida, India \\ ${ }^{2}$ Sapra Microbiology Centre, Gurugram, India \\ Email:u_sridhar@yahoo.com
}

How to cite this paper: Sridhar, U., Jain, P., Batra, J. and Sapra, N. (2017) Polymicrobial Keratitis-Importance of Detecting and Treating More Than One Organism. Open Journal of Ophthalmology, 7, 64-72. https://doi.org/10.4236/ojoph.2017.71010

Received: January 22, 2017

Accepted: February 20, 2017

Published: February 23, 2017

Copyright (c) 2017 by authors and Scientific Research Publishing Inc. This work is licensed under the Creative Commons Attribution International License (CC BY 4.0).

http://creativecommons.org/licenses/by/4.0/

\section{(c) (i) Open Access}

\begin{abstract}
Corneal ulcers may become non-healing if inappropriately managed. Empirically giving broad spectrum antibiotics or prescription of a "cocktail" of topical antibacterial, antifungal and antiviral medication may only add to drug toxicity and drug resistance. We present a series of four cases of microbial keratitis which are complicated due to presence of more than one causative organism. Microbiological work-up revealed Proteus species and fungal hyphae in smears only in the first case, atypical Mycobacteria and Staphyloccocus species in the second case, Moraxella and Streptococcus viridans in the third case and Aspergillus flavus and Nocardia species in the fourth case. Authors would like to emphasize the need for laboratory support in the treatment of corneal ulcers and importance of proper management of this sight threatening disease.
\end{abstract}

\section{Keywords}

Polymicrobial Keratitis, Broad Spectrum Antibiotics, Microbiological Work-Up

\section{Introduction}

Microbial keratitis is one of the main causes of corneal blindness especially in the developing country. Management involves identification of the causative organism and appropriate pharmacological therapy. However, either due to lack of laboratory support, or due to paucity of time in a busy clinic, availability of broad spectrum topical antibiotics or various other reasons, patient receives a "cocktail" of topical and systemic medications usually in inadequate dosages. This leads to the organism(s) developing resistance to the drug(s) and in addition, there is damage to the ocular surface due to drug toxicity.

The casual approach taken by many practitioners in the treatment of micro- 
bial keratitis may be the main reason for corneal ulcers becoming non-healing and may even lead eventually to complete loss of vision.

We present a series of non-healing microbial keratitis where proper microbiological work-up helps in detecting more than one organism in each of these ulcers as the causative factors.

\section{Case 1}

A young male immunocompetent doctor presented with redness, pain, watering and decrease in vision in his right eye since one month. He was being patched in that eye intermittently for a non healing epithelial defect since one ten days and in between was using Moxifloxacin eye drops four times a day and carboxy methyl cellulose $0.5 \%$ four times a day. On examination his BCVA was PL + PR accurate in his right eye and 6/6 in his left eye. Right eye showed mechanical ptosis, conjunctival and ciliary congestion. Cornea showed a small raised plaque like infiltrate $3 \times 3 \mathrm{~mm}$ covering the pupillary area with a small area of infiltrate around it. There was a $1 \mathrm{~m}$ hypopyon in the anterior chamber. No fundus view was possible. Ultrasound B scan showed anechoic vitreous cavity. The nasolacrimal passages were patent on both sides. Microbiological work up was done. On scraping the plaque lot of filamentous septate hyphae were seen on $10 \% \mathrm{KOH}$ mount (Figure 1(a)), Gram stain showed Gram negative bacilli which also grew on culture on blood and chocolate agar and was identified later as Proteus sps (Morganella). Fungus did not grow on culture media. The patient was given hourly natamycin $5 \%$ eye drops and initially Moxifloxacin $0.5 \%$ was given one hourly before sensitivity reports were received. After 3 days, the ulcer increased to $6 \times 5 \mathrm{~mm}$ in size (Figure $1(\mathrm{~b})$ ). It was re scraped and re cultured. Rescraping also showed fungal hyphae and gram negative bacilli which were reconfirmed as the same species of Proteus which was earlier grown in culture. $1 \%$ voriconazole eye drops hourly was added to the treatment regimen. The ulcer continued to increase in size and the edges were actively hyphate (Figure 2(a)). Sensitivity reports now came in and the treatment regimen was changed to $2.5 \%$ Amikacin

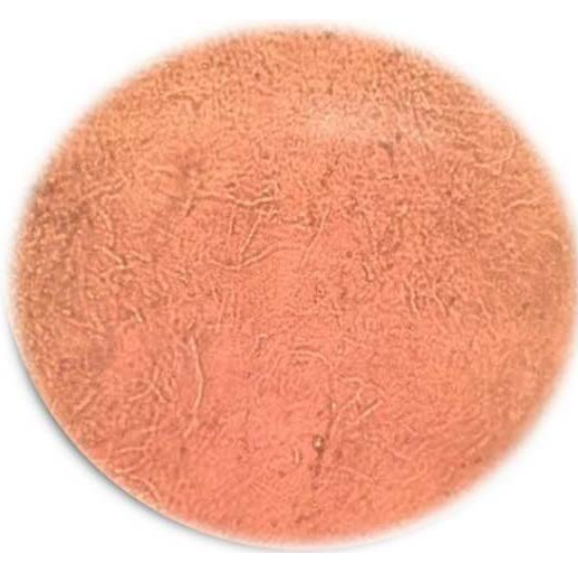

(a)

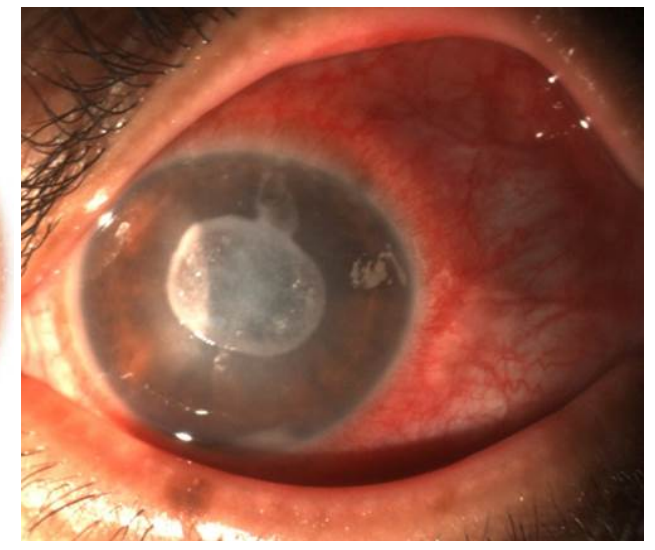

(b)

Figure 1. (a) case 1 fungal hyphae on $10 \% \mathrm{KOH}$ mount from corneal scraping on first day of presentation. (b) case 1 patient on day 3 plaque like infiltrate with hypopyon. 
one hourly and Fortified tobramycin one hourly along with topical Natamycin $5 \%$. As the response was slow and the patient was very uncomfortable on instillation of voriconazole eye drops, decision was taken to change it to topical $1 \%$ fluconazole eye drops. The cornea was showing extreme thinning (Figure 2(b)) which was documented by anterior segment optical coherence tomography. After 2 days, the patient came with a flat anterior chamber as the ulcer had perforated. Tissue adhesive and bandage contact lens application was done. Ulcer was showing scarring around the edges.

The ulcer showed further scarring around the edges with a descemetocele developing beyond the area of application of the tissue adhesive (Figure 3(a)). Anterior chamber remained well formed. The ulcer healed (Figure 3(b)) and UCVA was counting fingers close to face. Optical penetrating keratoplasty is now planned.

\section{Case 2}

A thirty-year-old immunocompetent female patient presented with decreased vision, pain and redness in her left eye since six weeks. She had been on treatment for corneal ulcer in her left eye since last ten days with hourly natamycin eye

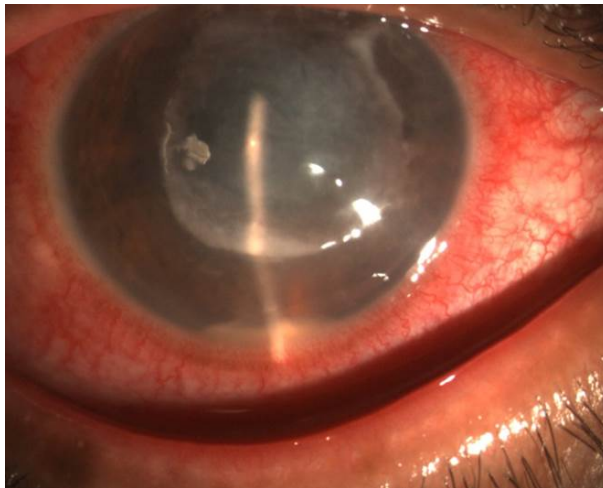

(a)

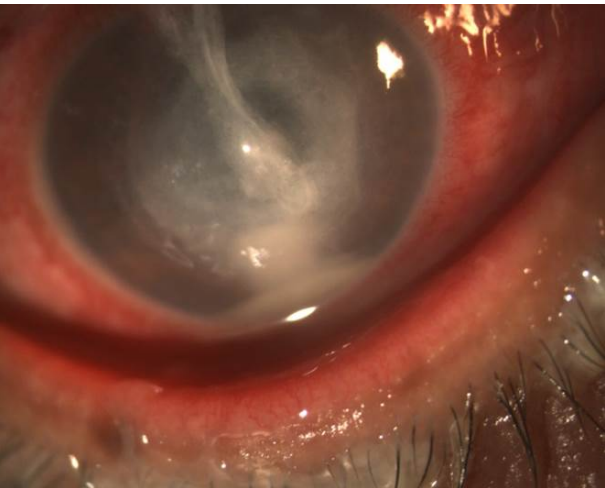

(b)

Figure 2. (a) case 1 worsening ulcer-size of infiltrate increased. (b) case 1 further worsening and thinning. Discharge seen on surface of cornea.

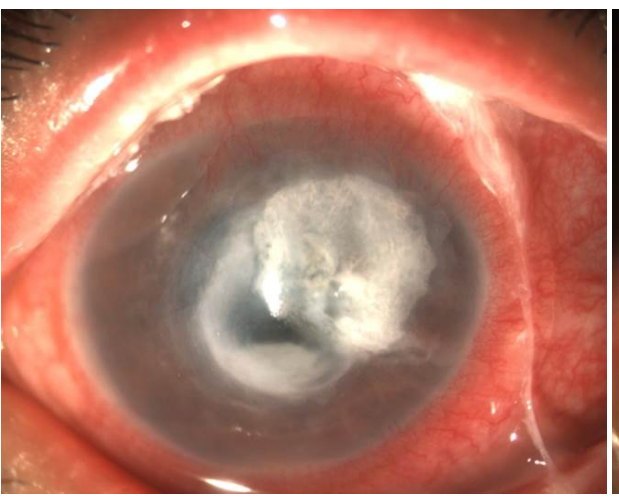

(a)

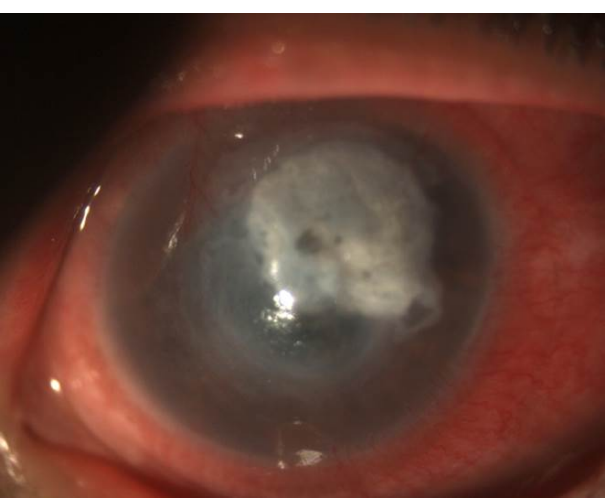

(b)

Figure 3. (a) case 1 glue + BCL application and further thinning beyond the glue. (b) healing of the ulcer. Glue is still on. 
drops; Moxifloxacin eye drops two hourly and cycloplegic eye drops. Prior to that, she had put medication in her eye which was prescribed by her village pharmacist, details of which she could not recollect. There was no history of trauma to her left eye or any foreign body falling into her left eye.

On examination, her BCVA in the right eye was 6/6 and HMCF in her left eye. Left eye showed conjunctival and ciliary congestion. Cornea showed a large infiltrate in the anterior and mid stroma extending in length almost from superior limbus to $4 \mathrm{~mm}$ short of inferior limbus. Width of infiltrate was variable, $3 \mathrm{~mm}$ wide in some places to $4-5 \mathrm{~mm}$ wide in the centre (Figure $4(\mathrm{a})$ and Figure 4(b)). There was hypopyon of $1.5 \mathrm{~mm}$ height in the anterior chamber. Microbiological workup was done twenty four hours after stopping all treatment. 10\% $\mathrm{KOH}$ mount and Gram stain did not show any fungal elements. Gram stain showed few Gram positive cocci and Gram variable coccobacilli. 20\% acid fast staining showed red colored bacilli (Figure 5). Coagulase negative Staphylococcus and Atypical Mycobacteria grew in culture (Figure 6). Based on sensitivity reports fortified Amikacin 2.5\% and fortified cefazoline 5\% was given one hourly. The infiltrate decreased in size and hypopyon reduced after a few days.

\section{Case 3}

A sixty-year-old male patient presented to our clinic with decreased vision in his

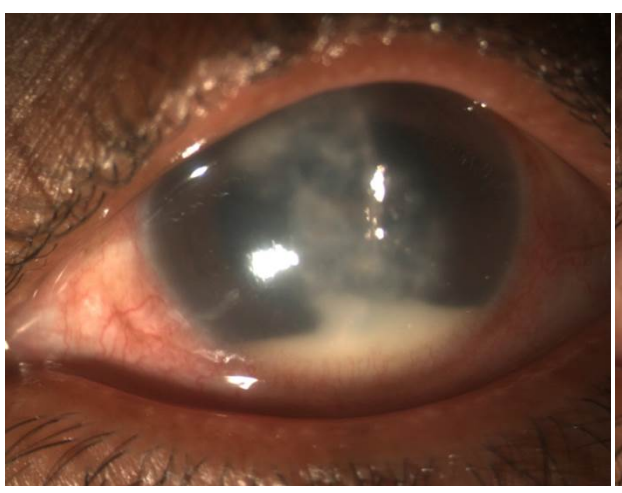

(a)

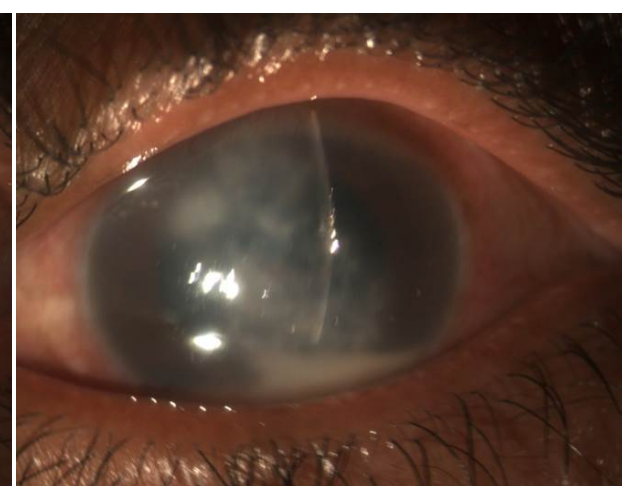

(b)

Figure 4. (a) case 2 stromal infiltrate with feathery margins and endoexudates. (b) case 2 slight decrease in hypopyon after starting treatment.

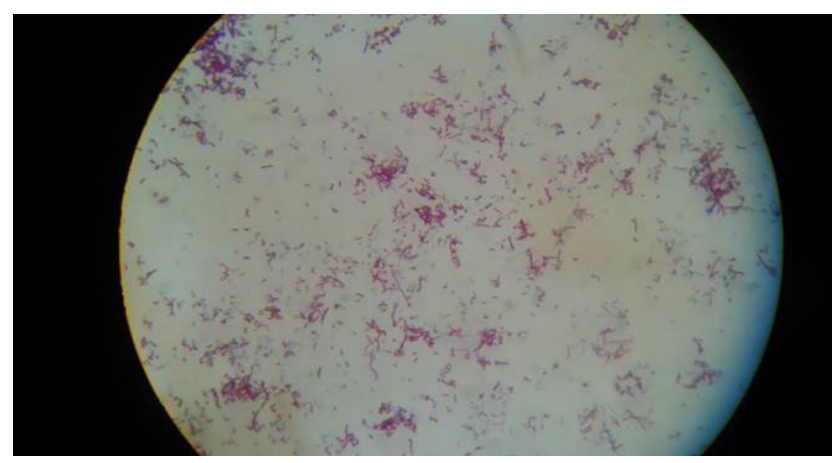

Figure 5. Case 2 Acid fast bacilli after decolorization by $20 \%$ sufuric acid. 
left eye since ten days. He was a farmer by profession. He had no history of trauma or foreign body falling in his left eye. He had lost vision in his right eye many years ago due to trauma. In the left eye too he had been treated for neurotrophic ulcer four years ago in our clinic with a lateral tarsorraphy and amniotic membrane graft. His BCVA in his right eye was no light perception and counting fingers half metre in his left eye. Left eye showed a lateral tarsorrhaphy in place. There was $3 \times 4 \mathrm{~mm}$ central corneal infiltrate which was anterior to mid stromal and a hypopyon $2 \mathrm{~mm}$ in height was present in the anterior chamber (Figure 7). Microbiological work up showed presence of Gram negative diplobacilli (Figure 8) and Gram positive cocci in pair and chains, Streptococcus viridians and Moraxella grew in culture. Based on smear, fortified amikacin 2.5\% and fortified cefazoline 5\% was started hourly. Patient returned ten days later with a perforation and a flat anterior chamber. Tissue adhesive and bandage contact lens was applied. The ulcer showed signs of healing after a week.

\section{Case 4}

A young immunocompetent male patient presented with history of redness,

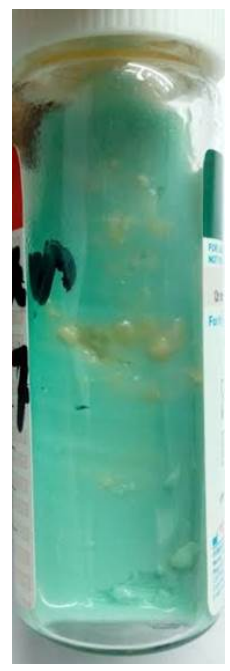

Figure 6. Case 2 Lowenstein-Jensens medium showing growth.

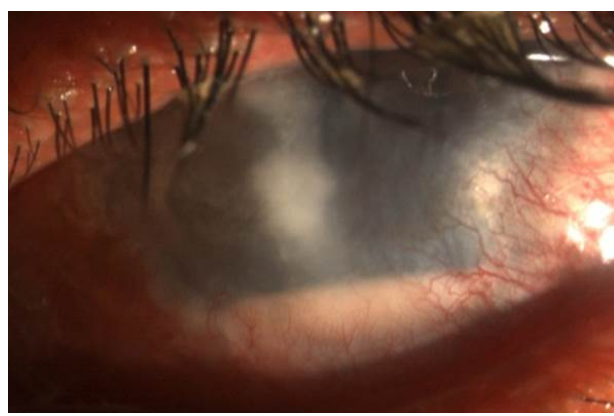

Figure 7. Case 3 stromal infiltrate with hypopyon in a patient who had tarsorrhaphy earlier for neurotrophic ulcer. 
pain, watering and decreased visual acuity in his right eye which was of ten days duration. He has been diagnosed as fungal keratitis elsewhere and treated with topical 5\% Natamycineye drops one hourly, topical Moxifloxacin $0.5 \%$ eye drops hourly. Microbiological work up done else where had shown fungal hyphae on $10 \% \mathrm{KOH}$ mount. On examination, BCVA was $6 / 24$ in the right eye and 6/6 in the left eye.

Right eye showed lid edema, conjunctival and ciliary congestion. Cornea showed a wet looking para central infiltrate $3 \times 3 \mathrm{~mm}$ size, anterior stromal in location, with surrounding corneal edema (Figure 9). Corneal scraping was done. $10 \% \mathrm{KOH}$ mount showed fungal hyphae. Gram stain showed presence of beaded filamentous bacilli and Gram variable cocobacillary forms. $1 \%$ acid fast stain was done and it showed red filaments and coccobacilli typical of Nocardia, Based on smear, topical fortified Cefazoline $5 \%$ hourly and fortified Tobramycin hourly was added to the regimen and topical Moxifloxacin was discontinued. Topical 5\% Natamycin was continued one hourly. Both organisms grew on culture medium. Aspergillus flavus and Nocardia sps (Figure 10) were the organisms identified.

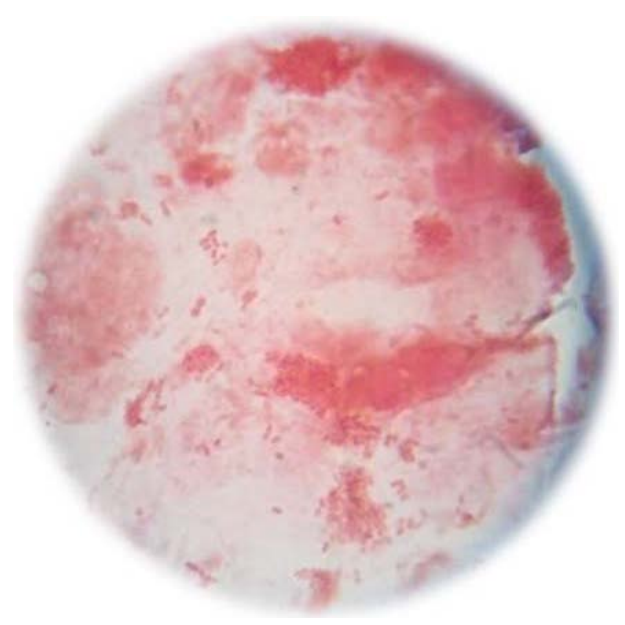

Figure 8. Case 3 Gram negative diplobacilli grown in culture-seen on Gram stain.

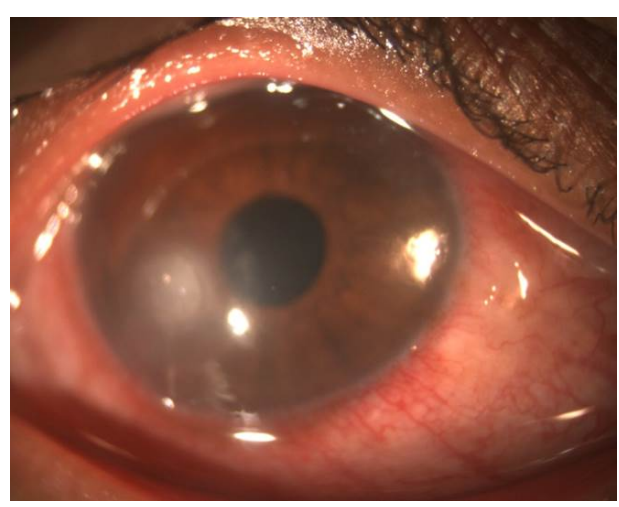

Figure 9. Case 4 stromal infiltrate with surrounding corneal edema in a patient on treatment with antibacterial and antifungals. 


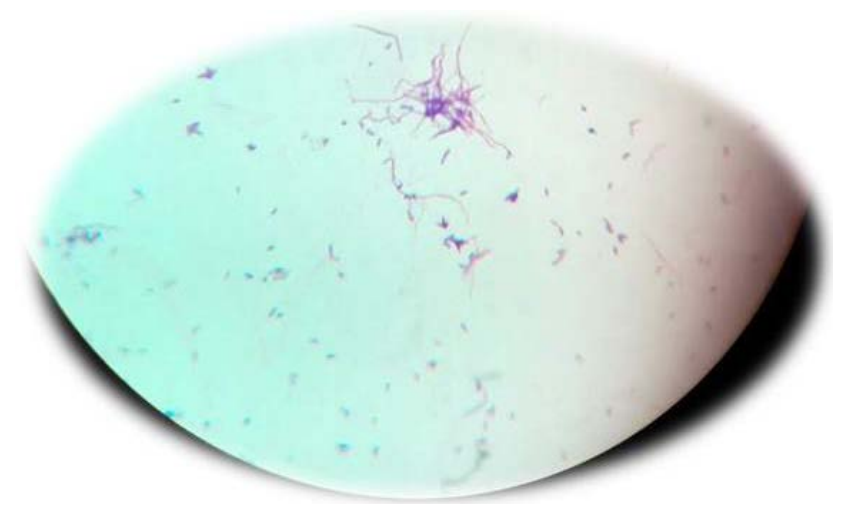

Figure 10. Case 4 acid fast bacilli on decolorization with $1 \%$ sulfuric acid.

The patient showed healing with scarring with the new treatment regimen.

\section{Discussion}

Poly microbial keratitis is not uncommonly encountered. Strong suspicion must be entertained under the following circumstances: slow or no healing with appropriate antimicrobial therapy or a previously responding corneal ulcer which suddenly worsens, as seen both symptomatically and clinically.

In a study by Fernandes [1] et al. compared clinical features and outcomes of polymicrobial keratitis where more than one organism grew in culture to cases where fungus alone was grown in culture. They found that the polymicrobial keratitis group of patients were older, (50.03 \pm 9.81 years vs. $42.79 \pm 12.15$ years, $\mathrm{P}=0.0038)$, with larger infiltrates at presentation $(61.8 \%$ vs. $24.1 \%, \mathrm{P}=0.0007)$, a higher association with endophthalmitis $(11.8 \%$ vs. $0 \%, \mathrm{P}=0.03)$, previous history of corneal graft $(20.6 \%$ vs. $0 \%, P=0.0012)$, and prior topical corticosteroid use $(23.5 \%$ vs. $5 \%, \mathrm{P}=0.019)$. In the polymicrobial group, a combination of bacteria and fungus was more frequently isolated $(23 \%, 67.6 \%)$, among which filamentous fungi $(25 \%, 39.1 \%)$ and coagulase-negative staphylococci $(14 \%$, 21.9\%) comprised a majority. Complete success defined as healing without perforation, was significantly lower in the polymicrobial group compared to the fungal keratitis group ( $39.3 \%$ vs. $73.7 \%, P=0.0045)$. In multivariate logistic regression analysis comparing factors affecting the outcome between the 2 groups, older age $(P=0.027)$ and ulcers larger than $6 \mathrm{~mm}(\mathrm{P}=0.001)$ at presentation adversely affected outcome. The authors concluded that polymicrobial keratitis with fungus and bacteria was more common and more challenging to treat, with a poorer outcome than fungal keratitis. Medical treatment may be effective; however, therapeutic penetrating keratoplasty (PKP) provided globe salvage at best. Early PKP may be advocated for larger ulcers at presentation.

A retrospective study by Lim et al. [2], found that the mean age of patients with polymicrobial keratitis was significantly higher than those with monomicrobial keratitis. Use of contact lens was the commonest predisposing factor in both groups. Systemic (23.8\%) and multiple (33.3\%) risk factors were involved in eyes with polymicrobial keratitis only. The mean size of corneal infiltrates and 
mean duration for resolvement of infection were significantly greater in the polymicrobial group. Medical treatment was successful only in $80.9 \%$ eyes with polymicrobial keratitis, whereas all monomicrobial keratitis patients responded to it. A total of 44 organisms belonging to 18 species (bacteria $=13$, fungi $=5$ ) were isolated from the polymicrobial group. P. aeruginosa and Candida albicans were the most frequently isolated bacteria $(n=12)$ and fungi $(n=5)$, respectively. In the polymicrobial group, gram-negative organisms were most sensitive to gentamicin $(87.8 \%)$, followed by ciprofloxacin $(78.7 \%)$, whereas gram-positive organisms were $100 \%$ sensitive to ciprofloxacin and cefazolin. The authors concluded that a high index of suspicion of polymicrobial keratitis should be made in patients with multiple and systemic risk factors. Contact lens usage was the most common risk factor in both groups. Size of corneal infiltrate is a fairly reliable indicator for suspecting polymicrobial keratitis. Prolonged course of the disease and decreased antibiotic sensitivity were the other notable features of polymicrobial keratitis.

In a review by Jones, [3] finding more than one isolate in a patient was attributed to these mechanisms 1) faulty technique or laboratory error, such as contamination of solid or liquid media during inoculation of corneal scrapings or processing in the laboratory, 2) colonization of the area of corneal ulceration by organisms present in the preocular tear film, 3) mixed infection by independent organisms, and 4) microbial synergism.

In our case series, patient age ranged from 32 to 70 years. Only one patient was 70 years old. All were immunocompetent. The seventy year male patient had history of neurotrophic ulcer treated by lateral tarsorrhaphy and amniotic membrane graft few years earlier and he was a farmer by profession. Case 1, patient was a doctor by profession, the infiltrate was treated by patching for ten days before he presented to us. Fungal hyphae were seen in smear and did not grow on culture. Proteus sps grew in culture. He responded to topical natamycin $\%, 1 \%$ fluconazole, $\mathrm{F}$ tobramycin and $\mathrm{f}$ amikacin $2.5 \%$. Perforation had to be treated by cyanocrylate adhesive and bandage contact lens application. Now optical PKP is planned for visual rehabilitation.

Case 2, young female patient, with no history of trauma, was under treatment with topical anti fungal and antimicrobial therapy since one month before presenting to us. Microbiological wok up pointed to infection with Streptococcus sps and atypical mycobacteria. Currently she is showing a slow response to treatment with topical fortified Tobramycin and $0.5 \%$ Moxifloxacin one hourly.

Case 3, was an elderly male patient who was one eyed. He was a farmer by profession and had been treated for neurotrophic ulcer by us few years earlier. He also had no history of trauma. Microbiological work up pointed to Moraxella a Gram negative diplobacillus and Gram positive cocci. F Cefazoline 5\% and F Amikacin 2.5\% helped to heal the ulcer. But perforation occurred which needed cyanoacrylate adhesive a bandage contact lens application. A small patch graft was done after complete healing of the ulcer.

Case 4 , was a young male patient, who was diagnosed as fungal ulcer and 
treated with topical antifungal agent (Natamycin 5\%) and topical Moxifoxacin $0.5 \%$ hourly. He suddenly had increase in pain and increase in size of ulceration which was earlier documented. Nocardia sps and Aspergillus flavus grew in culture. He responded well to topical fortified Cefazoline 5\% and topical Tobramycin $1.4 \%$ in addition to topical Natamycin $5 \%$.

In all these four cases more than one organism, fungus + bacteria (case 1 and 4) and two bacteria (case 2 and 4) were found. Fungus was filamentous septate hyaline and in case 4 grew in culture. Bacteria grown were Gram positive cocci (Streptococcus) typical Mycobacteria, Nocardiasps, Moraxella and Proteus sps. A larger incidence of Gram negative organisms was found.

All patients showed slow healing, case 1 and 4 perforated. Culture and sensitivity was done in all cases and helped to heal the lesions (case 1, 3, 4) and showed response in case 2.

Microbiological work-up is ulcers $>2 \mathrm{~mm}$ in size, and mandatory in nonhealing ulcers or those showing worsening after initial treatment response.

\section{Conclusion}

Polymicrobial keratitis is not uncommon. Diagnosis by means of corneal scraping and culture/sensitivity will aid in proper management of the lesions which will prove to be globe saving and sight saving.

\section{References}

[1] Fernandes, M., Vira, D., Dey, M., Tanzin, T., Kumar, N. and Sharma, S. (2015) Comparison between Polymicrobial and Fungal Keratitis: Clinical Features, Risk Factors, and Outcome. American Journal of Ophthalmology, 160, 873-881. https://doi.org/10.1016/j.ajo.2015.07.028

[2] Lim, N.C., Lim, D.K. and Ray, M. (2013) Polymicrobial versus Monomicrobial Keratitis: A Retrospective Comparative Study. Eye \& Contact Lens, 39, 348-354. https://doi.org/10.1097/ICL.0b013e3182a3024e

[3] Jones, D. (1981) Poly Microbial Keratitis. Transactions of the American Ophthalmological Society, 79.

\section{Scientific Research Publishing}

Submit or recommend next manuscript to SCIRP and we will provide best service for you:

Accepting pre-submission inquiries through Email, Facebook, LinkedIn, Twitter, etc. A wide selection of journals (inclusive of 9 subjects, more than 200 journals)

Providing 24-hour high-quality service

User-friendly online submission system

Fair and swift peer-review system

Efficient typesetting and proofreading procedure

Display of the result of downloads and visits, as well as the number of cited articles

Maximum dissemination of your research work

Submit your manuscript at: http://papersubmission.scirp.org/

Or contact ojoph@scirp.org 